\title{
COMPARISON THEOREMS FOR EIGENVALUE PROBLEMS FOR $n$ TH ORDER DIFFERENTIAL EQUATIONS
}

\author{
DARREL HANKERSON AND ALLAN PETERSON
}

(Communicated by Kenneth R. Meyer)

\begin{abstract}
We give a comparison theorem for eigenvalues for a $(k, n-k)$ conjugate boundary value problem for the systems $(-1)^{n-k} L y=\lambda P(t) y$ and $(-1)^{n-k} L z=\Lambda Q(t) z$, where $P(t)$ and $Q(t)$ are continuous $m \times m$ matrix functions. We assume that the corresponding scalar equation $L x=0$ is $(j, n-$ $j$ )-disconjugate for $k-1 \leq j \leq n-1$. A special case of this is when $L x=0$ is disconjugate; our results are new even in this case.
\end{abstract}

1. Introduction. We are concerned with proving comparison theorems for the eigenvalue problems

$$
(-1)^{n-k} L y=\lambda P(t) y
$$

and

$$
(-1)^{n-k} L z=\Lambda Q(t) z
$$

subject to the boundary conditions

$$
\begin{array}{ll}
y^{(i)}(a)=0, & 0 \leq i \leq k-1, \\
y^{(i)}(b)=0, & 0 \leq i \leq n-k-1 .
\end{array}
$$

Here, $1 \leq k \leq n-1, P$ and $Q$ are continuous $m \times m$ matrix functions on $[a, b]$, and

$$
L y(t)=\sum_{i=0}^{n} a_{i}(t) y^{(i)}(t)
$$

where $a_{i}(t), 0 \leq i \leq n$, are continuous scalar functions on $[a, b]$ with $a_{n}(t)>0$ on $[a, b]$.

In this paper, we prove existence and comparison theorems for the least positive eigenvalues of (1), (3) and (2), (3), respectively. The methods used depend on the theory of operators on a cone; the books of Krasnosel'skii [6] and Deimling [1] are good references. Related papers include those of Gentry and Travis [2], Keener and Travis [4, 5], Kreith [7], Schmitt and Smith [10], Smith [11], Tomastik [13], and the authors [3].

Received by the editors September 3, 1987 and, in revised form, January 4, 1988.

1980 Mathematics Subject Classification (1985 Revision). Primary 34C10; Secondary 34B05, 34B10.

Key words and phrases. Comparison theorem, boundary value problem, $u_{0}$-positive operator, disconjugacy. 
Typically, "positivity" conditions are placed on $P(t)$ and $Q(t)$ in order that related operators be $u_{0}$-positive. This leads to existence and comparison theorems for the least positive eigenvalues. In addition, we show how these requirements can be relaxed and still obtain existence and comparison results.

2. Auxiliary results. We say that the scalar equation

$$
L x \equiv \sum_{i=0}^{n} a_{i}(t) x^{(i)}=0
$$

is $(j, n-j)$-disconjugate on $[a, b]$ provided there is no nontrivial solution $x(t)$ and numbers $a \leq \alpha<\beta \leq b$ such that

$$
\begin{array}{ll}
x^{(i)}(\alpha)=0, & 0 \leq i \leq j-1, \\
x^{(i)}(\beta)=0, & 0 \leq i \leq n-j-1 .
\end{array}
$$

We say that $L x=0$ is disconjugate on an interval $I$ if no nontrivial solution has $n$ or more zeros (counting multiplicities) on $I$.

It is well known [8] that $L x=0$ is $(j, n-j)$-disconjugate on an interval $I$ if $L$ is of the form

$$
L x=M x+r(t) x, \quad(-1)^{n-j} r(t) \geq 0 \quad \text { on } I
$$

where $M x=0$ is any $n$th order linear disconjugate equation on $I$ (with leading coefficient positive).

We will assume that the scalar equation $L x=0$ is $(j, n-j)$-disconjugate on $[a, b]$ for $j=k-1, \ldots, n-1$ (if $k=1$, we assume $L x=0$ is disconjugate on $[a, b]$ ). It is well known that if $L x=0$ is disconjugate on $[a, b]$ then this condition is satisfied. If $k>2$, then the converse need not hold. Our results appear to be new even in the disconjugate case. By Theorem 11 of [9], it follows that the Green's function $G(t, s)$ for the scalar problem

$$
\begin{aligned}
(-1)^{n-k} L x & =h(t) \\
x^{(i)}(a) & =0, \quad 0 \leq i \leq k-1, \\
x^{(i)}(b) & =0, \quad 0 \leq i \leq n-k-1,
\end{aligned}
$$

exists, and satisfies the positivity condition

$$
G(t, s)>0, \quad t, s \in(a, b) .
$$

We now give several definitions as given in [6] which we will use in this paper. Let $B$ be a Banach space. A closed nonempty subset $P$ of $B$ is said to be a cone provided

(i) if $u, v \in \mathcal{P}$ then $\alpha u+\beta v \in \mathcal{P}$ for all $\alpha, \beta \geq 0$,

(ii) if $u,-u \in P$ then $u=0$.

A cone $P$ is said to be reproducing provided every $w \in B$ can be written $w=u-v$ for some $u, v \in P$. A cone $P$ is solid provided its interior $P^{\circ}$ is nonempty. Every solid cone is reproducing (see [6]). If $P$ is a cone and $u, v \in B$ then we write $u \leq v$ with respect to $P$ provided $v-u \in P$. If $M$ and $N$ are operators on $B$, then we write $M \leq N$ with respect to $P$ provided $M u \leq N u$ for all $u \in P$. Finally we say that a bounded linear operator $M$ on $B$ is $u_{0}$-positive with respect to the cone $P$ 
provided $u_{0} \in P$ and for every nonzero $u \in P$ there exist positive numbers $k_{1}$ and $k_{2}$ (which in general depend on $u$ ) such that $k_{1} u_{0} \leq M u \leq k_{2} u_{0}$ with respect to $P$.

We will make use of the following results which we state here for easy reference. The first is Theorem 2.5 of Krasnosel'skiu [6].

THEOREM 1. Let $L$ be a linear compact operator which leaves the cone $P$ invariant. Assume there is $a u \in B$ and an $\varepsilon>0$ with $-u \notin P$ and $u=v-w$ for some $v, w \in P$ such that $L^{p} u \geq \varepsilon u$ for some natural number $p$. Then $L$ has at least one eigenvector $x_{0}$ in $P: L x_{0}=\lambda_{0} x_{0}$, where the eigenvalue $\lambda_{0}$ satisfies $\lambda_{0} \geq \sqrt[p]{\varepsilon}$.

COROLlaRY 1. Assume in addition to the hypotheses of Theorem 1 that $P$ is reproducing. Then one of the eigenvalues of $L$ corresponding to an eigenvector in $P$ is an upper bound for the modulus of the eigenvalues of $L$.

Theorems 2.5, 2.10, 2.11, and 2.13 of Krasnosel'skir [6] give the following theorem.

THEOREM 2. Let $P$ be a reproducing cone. If $L$ is a compact $u_{0}$-positive linear operator then $L$ has an essentially unique eigenvector in $P$ and the corresponding eigenvalue is simple, positive, and larger than the modulus of any other eigenvalue.

The following theorem appears in Keener and Travis [4, Theorem 2.3] and is a generalization of Theorem 2.3 of Travis [14].

THEOREM 3. Let $L$ and $M$ be bounded linear operators and assume that at least one of the operators is $u_{0}$-positive. If $L \leq M$ and

$$
\begin{array}{cl}
L u_{1} \geq \lambda_{1} u_{1} & \left(0 \neq u_{1} \in \mathcal{P}, \lambda_{1}>0\right), \\
M u_{2} \leq \lambda_{2} u_{2} & \left(0 \neq u_{2} \in \mathcal{P}, \lambda_{2}>0\right),
\end{array}
$$

then $\lambda_{1} \leq \lambda_{2}$, and if $\lambda_{1}=\lambda_{2}$ then $u_{1}$ is a scalar multiple of $u_{2}$.

For related statements of these results of Krasnosel'skil̆, see Theorems 2.1-2.4 $[\mathbf{1 0}]$.

3. Main results. Define the Banach space $B$ by

$$
\begin{aligned}
B=\left\{y \in C^{n}\left([a, b], R^{m}\right): y^{(i)}(a)=0,0 \leq i \leq k-1,\right. \\
\left.\qquad y^{(i)}(b)=0,0 \leq i \leq n-k-1\right\}
\end{aligned}
$$

where

$$
\|y\|=\max \left\{\left|y^{(i)}(t)\right|: t \in[a, b], 0 \leq i \leq n\right\}
$$

and $|\cdot|$ is the Euclidean norm. Let $K$ be a solid cone in $R^{m}$, and define the cone $P$ in $B$ by

$$
P=\{y \in B: y(t) \in K, a \leq t \leq b\}
$$

Except for a few of our general results, we will be interested in the special type of solid cone $K_{1}$ defined below. As in Tomastik's paper [13], let $I, J \subseteq\{1, \ldots, m\}$ be such that $I \cup J=\{1, \ldots, m\}$ and $I \cap J=\varnothing(I=\varnothing$ or $J=\varnothing$ is permissible $)$. Define the cone $K_{1} \subseteq R^{m}$ by

$$
\mathcal{K}_{1}=\left\{y=\left(y_{1}, \ldots, y_{m}\right): y_{i} \geq 0 \text { if } i \in I, y_{i} \leq 0 \text { if } i \in J\right\}
$$


Throughout this paper assume $\delta_{i}=1$ if $i \in I$, and $\delta_{i}=-1$ if $i \in J$. Note that $y \in K_{1}$ iff $\delta_{i} y_{i} \geq 0,0 \leq i \leq m$. Then define the cone $P_{1}$ in $B$ by

$$
P_{1}=\left\{y \in B: y(t) \in K_{1}, t \in[a, b]\right\} .
$$

We remark that it is not true that the interior of the cone $P$ is the set $\{y \in$ $B: y(t) \in \mathcal{K}^{\circ}$ for $\left.t \in(a, b)\right\}$, although this type of oversight appears in some of the literature. Keep this in mind when reading the proof of Theorem 4 below.

Define operators $M$ and $N$ on $B$ by

$$
\begin{array}{ll}
M u(t)=\int_{a}^{b} G(t, s) P(s) u(s) d s, & t \in[a, b], \\
N u(t)=\int_{a}^{b} G(t, s) Q(s) u(s) d s, & t \in[a, b] .
\end{array}
$$

It is clear that $M$ and $N$ are linear, and a standard argument using Ascoli's Theorem shows that they are compact operators.

REMARK 1. If $\lambda \neq 0$ is an eigenvalue of $M$ with corresponding eigenvector $y$, then $y$ is an eigenfunction of $(1),(3)$ with corresponding eigenvalue $1 / \lambda$. Note that $\lambda=0$ is not an eigenvalue for (1),.(3) since $L x=0$ is $(k, n-k)$-disconjugate on $[a, b]$.

THEOREM 4. Assume $Q(t) \mathcal{K} \subseteq \mathcal{K}$ for $t \in[a, b]$, and for each nontrivial $u$ in $P$, there exists $t_{u} \in[a, b]$ such that $Q\left(t_{u}\right) u\left(t_{u}\right) \in \mathcal{K}^{\circ}$. Then the boundary value problem (2), (3) has a smallest positive eigenvalue $\Lambda_{0}$, and $\Lambda_{0}$ is smaller than the modulus of any other eigenvalue of (2), (3). Further, there is an essentially unique eigenfunction $z_{0}(t)$ corresponding to $\Lambda_{0}$ and either $z_{0} \in \mathcal{P}^{\circ}$ or $-z_{0} \in \mathcal{P}^{\circ}$.

Proof. We will show that $N: P \backslash\{0\} \rightarrow P^{\circ}$. Let $0 \neq u \in P$ and define $h$ by

$$
h(t)=N u(t)=\int_{a}^{b} G(t, s) Q(s) u(s) d s .
$$

It follows (see Lemma 2.3 of [5]) from (4) and the assumptions on $Q(t)$ that

$$
h(t) \in \mathcal{K}^{\circ}, \quad t \in(a, b) \text {. }
$$

As we pointed out earlier, this does not imply that $h \in \mathcal{P}^{\circ}$. To see that $h \in \mathcal{P}^{\circ}$, we will assume that $h \notin P^{\circ}$ and show that this leads to a contradiction.

Since $h \notin \mathcal{P}^{\circ}$, there is a sequence $\left\{y^{j}\right\} \subseteq B$ such that $y^{j} \rightarrow h$ in $B$ and $y^{j} \notin P$, $j \geq 1$. Then there is a $t_{j} \in[a, b]$ such that $y^{j}\left(t_{j}\right) \notin K$ for each $j \geq 1$. Without loss of generality, we can assume $t_{j} \rightarrow t_{0}$ where $t_{0} \in[a, b]$. If $t_{0} \in(a, b)$, then $y^{j}\left(t_{j}\right) \rightarrow h\left(t_{0}\right) \in \mathcal{K}^{\circ}$ implies that for sufficiently large $j, y^{j}\left(t_{j}\right) \in \mathcal{K}^{\circ}$ which is a contradiction.

The only possibilities that remain are that $t_{0}=a$ or $t_{0}=b$. We now use two other (in addition to (4)) very important properties of $G(t, s)$ which were established in [9]; these are

$$
\begin{aligned}
& G^{(k)}(a, s)>0, \quad s \in[a, b), \\
& (-1)^{n-k} G^{(n-k)}(b, s)>0, \quad s \in(a, b] \text {. }
\end{aligned}
$$

Since

$$
h^{(k)}(a)=\int_{a}^{b} G^{(k)}(a, s) Q(s) u(s) d s
$$


it follows from (5) that

$$
h^{(k)}(a) \in \mathcal{K}^{\circ}
$$

Similarly, using (6) it follows that

$$
(-1)^{n-k} h^{(n-k)}(b) \in \mathcal{K}^{\circ} .
$$
that

By Taylor's theorem about $t=a$ applied to the components $y_{i}^{j}$ of $y^{j}$, we get

where

$$
y^{j}\left(t_{j}\right)=\left(y^{j}\right)^{(k)}(a) \frac{\left(t_{j}-a\right)^{k}}{k !}+R\left(\xi_{1 j}, \ldots, \xi_{m j}\right) \frac{\left(t_{j}-a\right)^{k+1}}{(k+1) !}
$$

$$
R\left(\xi_{1 j}, \ldots, \xi_{m j}\right)=\left(\begin{array}{c}
\left(y_{1}^{j}\right)^{(k+1)}\left(\xi_{1 j}\right) \\
\vdots \\
\left(y_{m}^{j}\right)^{(k+1)}\left(\xi_{m j}\right)
\end{array}\right)
$$

where $a<\xi_{i j}<t_{j}, 1 \leq i \leq m, j \geq 1$. Since

$$
y^{j}\left(t_{j}\right)=\frac{\left(t_{j}-a\right)^{k}}{k !}\left[\left(y^{j}\right)^{(k)}(a)+R\left(\xi_{1 j}, \ldots, \xi_{m j}\right) \frac{\left(t_{j}-a\right)}{k+1}\right],
$$

$\left(y^{j}\right)^{(k)}(a) \rightarrow h^{(k)}(a) \in \mathcal{K}^{\circ}$, and $R\left(\xi_{1 j}, \ldots, \xi_{m j}\right) \rightarrow h^{(k+1)}(a)$ as $j \rightarrow \infty$, it follows that $y^{j}\left(t_{j}\right) \in \mathcal{K}^{\circ}$ for $j$ sufficiently large, which is a contradiction. Hence $t_{0} \neq a$.

Similarly, if $t_{0}=b$, then we use (8) and apply Taylor's theorem to the components of $y^{j}$ to get a similar contradiction. Hence $N: P \backslash\{0\} \rightarrow P^{\circ}$. (Note that if we did not assume the zero conditions at $b$ in the definition of $B$, then our proof of $N: P \backslash\{0\} \rightarrow P^{\circ}$ breaks down here.)

Since $N: P \backslash\{0\} \rightarrow P^{\circ}, P^{\circ}$ is nonempty. Therefore $P$ is a solid cone and hence a reproducing cone in $B$. Pick $u_{0} \in P^{\circ}$. Then $N: P \backslash\{0\} \rightarrow P^{\circ}$ implies $N$ is $u_{0}$-positive with respect to $P$. The proof of this last statement appears in [5, p. 253]. For completeness we repeat this argument here. Since $N u \in \mathcal{P}^{\circ}$, we have for sufficiently small $k_{1}>0$ that $N u-k_{1} u_{0} \in P$. On the other hand $u_{0} \in P^{\circ}$ so for $k_{2}>0$ sufficiently large, $u_{0}-N u / k_{2} \in P$. It follows that $k_{1} u_{0} \leq N u \leq k_{2} u_{0}$ with respect to $P$, and so $N$ is a $u_{0}$-positive operator. The conclusions of this theorem then follow from Theorem 2 and the $u_{0}$-positivity of $N$.

COROLLARY 2. If for $1 \leq i, j \leq m$,

$$
q_{i j}(t) \delta_{i} \delta_{j} \geq 0, \quad t \in[a, b],
$$

and $q_{i j}$ is not identically zero on any subinterval of $[a, b]$, then the boundary value problem (2), (3) has a smallest positive eigenvalue $\Lambda_{0}$, and $\Lambda_{0}$ is smaller than the modulus of any other eigenvalue of (2), (3). Further, there is an essentially unique eigenfunction $z_{0}(t)$ corresponding to $\Lambda_{0}$ and either $z_{0}$ or $-z_{0}$ is in $P_{1}^{\circ}$. Hence, without loss of generality

$$
\delta_{i}\left(z_{0}(t)\right)_{i}>0, \quad t \in(a, b), 1 \leq i \leq m,
$$

where $\left(z_{0}(t)\right)_{i}$ is the ith component of $z_{0}(t)$.

ProOF. We will apply the above theorem with $K=K_{1}$ and $P=P_{1}$. It suffices to show that $Q(t) K_{1} \subseteq \mathcal{K}_{1}$ for $t \in[a, b]$, and for each $0 \neq u \in P_{1}$, there exists $t_{u} \in[a, b]$ such that $Q\left(t_{u}\right) u\left(t_{u}\right) \in \mathcal{K}_{1}^{\circ}$. 
Let $v \in K_{1}$. Then $\delta_{i} v_{i} \geq 0,1 \leq i \leq m$, and hence

$$
\delta_{i}(Q(t) v)_{i}=\sum_{j=1}^{m} \delta_{i} \delta_{j} q_{i j}(t) \delta_{j} v_{j} \geq 0, \quad t \in[a, b], 1 \leq i \leq m .
$$

Hence $Q(t) \mathcal{K}_{1} \subseteq \mathcal{K}_{1}$. If $0 \neq u \in \mathcal{P}_{1}$, then there is a $t_{1} \in[a, b]$ and a $j_{0} \in\{1, \ldots, m\}$ such that $\delta_{j_{0}} u_{j_{0}}\left(t_{1}\right)>0$. Pick $t_{u}$ sufficiently close to $t_{1}$ such that $\delta_{j_{0}} u_{j_{0}}\left(t_{u}\right)>0$ and $\delta_{i} \delta_{j_{0}} q_{i j_{0}}\left(t_{u}\right)>0,1 \leq i \leq m$. Then

$$
\begin{aligned}
\delta_{i}\left(Q\left(t_{u}\right) u\left(t_{u}\right)\right)_{i} & =\sum_{j=1}^{m} \delta_{i} \delta_{j} q_{i j}\left(t_{u}\right) \delta_{j} u_{j}\left(t_{u}\right) \\
& \geq \delta_{i} \delta_{j_{0}} q_{i j_{0}}\left(t_{u}\right) \delta_{j_{0}} u_{j_{0}}\left(t_{u}\right)>0
\end{aligned}
$$

for $1 \leq i \leq m$. It follows that $Q\left(t_{u}\right) u\left(t_{u}\right) \in \mathcal{K}_{1}^{\circ}$ and the proof is complete.

THEOREM 5. Assume $P(t)$ and $Q(t)$ satisfy the hypothesis of Theorem 4 and that $P(t) \leq Q(t)$ with respect to $\mathcal{K}, t \in[a, b]$. Then the smallest positive eigenvalues $\lambda_{0}$ and $\Lambda_{0}$ of (1), (3) and (2), (3), respectively, satisfy $\Lambda_{0} \leq \lambda_{0}$. Furthermore, if $\Lambda_{0}=\lambda_{0}$ then

$$
P(t) z_{0}(t)=Q(t) z_{0}(t), \quad t \in[a, b],
$$

where $z_{0}(t)$ is as in Theorem 4.

Proof. By the proof of Theorem $4, M$ and $N$ are $u_{0}$-positive with respect to $P$. Further, by Theorem 4, the smallest positive eigenvalues $\lambda_{0}$ and $\Lambda_{0}$ exist.

It is easy to see that $P(t) \leq Q(t)$ with respect to $\mathcal{K}, t \in[a, b]$, implies that $M \leq N$ with respect to $P$. The conclusions of this theorem follow easily from Theorem 3.

For the cone $K_{1}$, we can weaken the conditions on $P(t)$ by employing Theorem 1 and Corollary 1. This will enable us to prove more general results. Even in the case $L x=x^{(n)}$ we believe that these results are new.

LEMMA 1. Assume

$$
p_{i j}(t) \delta_{i} \delta_{j} \geq 0, \quad t \in[a, b],
$$

$1 \leq i, j \leq m$, and there is a $t_{0} \in[a, b]$ and an $i_{0} \in\{1, \ldots, m\}$ such that

$$
p_{i_{0} i_{0}}\left(t_{0}\right)>0 \text {. }
$$

Then the eigenvalue problem (1), (3) has a least positive eigenvalue $\lambda_{0}$ which is a lower bound on the modulus of all eigenvalues of (1), (3). Furthermore, there is an eigenfunction $y_{0}(t)$ corresponding to $\lambda_{0}$ such that

$$
\delta_{i}\left(y_{0}(t)\right)_{i} \geq 0, \quad t \in[a, b], 1 \leq i \leq m .
$$

ProOF. Let $u \in P_{1}$ and consider

$$
M u(t)=\int_{a}^{b} G(t, s) P(s) u(s) d s
$$

It follows that

$$
\delta_{i}(M u)_{i}(t)=\int_{a}^{b} G(t, s) \sum_{j=1}^{m} \delta_{i} \delta_{j} p_{i j}(s) \delta_{j} u_{j}(s) d s .
$$


Hence

$$
\delta_{i}(M u)_{i}(t) \geq 0, \quad t \in[a, b], 1 \leq i \leq m,
$$

and we have that $M: P_{1} \rightarrow P_{1}$.

In order to apply Theorem 1, we now show that there is a $w \in P_{1}$ and an $\varepsilon_{0}>0$ such that $M w \geq \varepsilon_{0} w$ with respect to the cone $P_{1}$. To this end, define $w$ by

$$
w(t)=(t-a)^{k}(b-t)^{n-k} \delta_{i_{0}} e_{i_{0}}, \quad a \leq t \leq b,
$$

where $e_{i_{0}}$ is the unit vector in $R^{m}$ in the $i_{0}$ direction. Note that

$$
\delta_{i_{0}}(M w)_{i_{0}}(t)=\int_{a}^{b} G(t, s) \delta_{i_{0}} \delta_{i_{0}} p_{i_{0} i_{0}}(s)(s-a)^{k}(b-s)^{n-k} d s>0
$$

for $t \in(a, b)$ follows from (9) and (10). Using (5) and (6), it is easy to show that

$$
\begin{aligned}
\delta_{i_{0}}(M w)_{i_{0}}^{(k)}(a) & >0, \\
(-1)^{n-k} \delta_{i_{0}}(M w)_{i_{0}}^{(n-k)}(b) & >0 .
\end{aligned}
$$

Hence $(M w)_{i_{0}}(t)$ has a zero of exact order $k$ at $a$ and a zero of exact order $n-k$ at $b$. But $w_{i_{0}}(t)$ satisfies

$$
\begin{aligned}
& \delta_{i_{0}} w_{i_{0}}(t)>0, \quad t \in(a, b), \\
& w_{i_{0}}^{(i)}(a)=0, \quad 0 \leq i \leq k-1, \\
& w_{i_{0}}^{(i)}(b)=0, \quad 0 \leq i \leq n-k-1, \\
& \delta_{i_{0}} w_{i_{0}}^{(k)}(a)>0, \quad(-1)^{n-k} \delta_{i_{0}} w_{i_{0}}^{(n-k)}(b)>0 .
\end{aligned}
$$

It follows that for $\varepsilon_{0}>0$ sufficiently small

$$
\delta_{i_{0}}(M w)_{i_{0}}(t) \geq \varepsilon_{0} \delta_{i_{0}} w_{i_{0}}(t), \quad t \in[a, b] .
$$

Then it is easy to see that $M w \geq \varepsilon_{0} w$ with respect to the cone $P_{1}$. By Theorem 1 , Corollary 1 , and Remark 1 , the conclusions of this lemma follow.

THEOREM 6. In addition to the assumption that the scalar equation $L x=0$ is $(j, n-j)$-disconjugate on $[a, b], k-1 \leq j \leq n-1$, assume

(a) there is an $i_{0} \in\{1, \ldots, m\}$ and $t_{0} \in[a, b]$ such that $p_{i_{0} i_{0}}\left(t_{0}\right)>0$,

(b) $0 \leq p_{i j}(t) \delta_{i} \delta_{j} \leq q_{i j}(t) \delta_{i} \delta_{j}, t \in[a, b], 1 \leq i, j \leq m$, and $q_{i j} \not \equiv 0$ on any (nondegenerate) subinterval of $[a, b], 1 \leq i, j \leq m$.

Then there exists a smallest positive eigenvalue $\lambda_{0}$ (which is a lower bound for the modulus of the eigenvalues) of (1), (3) and a corresponding eigenvector $y_{0}(t)$ where $y_{0} \in P_{1}$. The eigenvalue problem (2), (3) has a smallest positive eigenvalue $\Lambda_{0}$ (which is less than the modulus of any other eigenvalue of (2), (3)) and a corresponding essentially unique eigenfunction $z_{0}(t)$. We can pick $z_{0} \in \mathcal{P}_{1}^{\circ}$, and then $z_{0}(t)$ satisfies

$$
\delta_{i}\left(z_{0}\right)_{i}(t)>0, \quad t \in(a, b), 1 \leq i \leq m .
$$

Furthermore, $\Lambda_{0} \leq \lambda_{0}$, and $\Lambda_{0}=\lambda_{0}$ iff $P(t)=Q(t)$ for $t \in[a, b]$.

Proof. By Lemma $1, \lambda_{0}$ and $y_{0}$ exist as in the statement of the theorem. By Corollary 2, the eigenvalue problem (2), (3) has a smallest positive eigenvalue $\Lambda_{0}$ and corresponding to $\Lambda_{0}$ there is an essentially unique eigenvector $z_{0}$, and either 
$z_{0} \in P_{1}$ or $-z_{0} \in P_{1}$. Pick $z_{0} \in P_{1}$. It can be shown that $M \leq N$. Hence by Theorem 3, we get that $\Lambda_{0} \leq \lambda_{0}$.

Now assume $\Lambda_{0}=\lambda_{0}$. Then by Theorem $3, y_{0}(t)$ and $z_{0}(t)$ are linearly dependent on $[a, b]$. It follows from (1) and (2) that

$$
P(t) z_{0}(t)=Q(t) z_{0}(t), \quad t \in[a, b] .
$$

Hence for $1 \leq i \leq m$,

$$
\sum_{j=1}^{m}\left[q_{i j}(t)-p_{i j}(t)\right]\left(z_{0}\right)_{j}(t)=0, \quad t \in[a, b] .
$$

Then

$$
\sum_{j=1}^{m}\left[\left(q_{i j}(t)-p_{i j}(t)\right) \delta_{i} \delta_{j}\right] \delta_{j}\left(z_{0}\right)_{j}(t)=0 .
$$

Since $z_{0}$ satisfies (11) and condition (b) holds, we get that

$$
p_{i j}(t)=q_{i j}(t), \quad t \in(a, b), 1 \leq i, j \leq m .
$$

By continuity, it follows that $P(t)=Q(t)$ on the closed interval $[a, b]$.

\section{REFERENCES}

1. K. Deimling, Nonlinear functional analysis, Springer-Verlag, 1985.

2. R. D. Gentry and C. C. Travis, Comparison of eigenvalues associated with linear differential equations of arbitrary order, Trans. Amer. Math. Soc. 223 (1976), 167-179.

3. D. Hankerson and A. Peterson, Comparison of eigenvalues for focal point problems for $n$th order difference equations, (submitted).

4. M. S. Keener and C. C. Travis, Positive cones and focal points for a class of $n$th order differential equations, Trans. Amer. Math. Soc 237 (1978), 331-351.

5. $\ldots$ Sturmian theory for a class of nonselfadjoint differential systems, Ann. Mat. Pura Appl. 123 (1980), 247-266.

6. M. A. Krasnosel'skiř, Positive solutions of operator equations, Fizmatgiz, Moscow, 1962; English transl., Noordhoff, Groningen, 1964.

7. K. Kreith, A class of hyperbolic focal point problems, Hiroshima Math. J. 14 (1984), 203-210.

8. A. Ju. Levin, Distribution of the zeros of solutions of a linear differential equation, Soviet Math. Dokl. 5 (1964), 818-821.

9. A. Peterson, On the sign of Green's functions, J. Differential Equations 21 (1976), 167-178.

10. K. Schmitt and H. L. Smith, Positive solutions and conjugate points for systems of differential equations, Nonlinear Anal. 2 (1978), 93-105.

11. H. Smith, A note on disconjugacy of second order systems, Pacific J. Math. 89 (1980), 447-452.

12. E. Tomastik, Comparison theorems for second order nonselfadjoint differential systems, SIAM J. Math. Anal. 14 (1983), 60-65.

13. Comparison theorems for conjugate points of $n$th order nonselfadjoint differential equations, Proc. Amer. Math. Soc. 96 (1986), 437-442.

14. C. C. Travis, Comparison of eigenvalues for linear differential equations of order $2 n$, Trans. Amer. Math. Soc. 177 (1973), 363-374.

Department of Algebra, Combinatorics and Analysis, Auburn University, Auburn, Alabama 36849

Department of Mathematics and Statistics, University OF NEbraskaLINCOLN, LINCOLN, NEBRASKA 68588 